underwent LLETZ. All women who had initial successful treatment were included in further analysis.

Follow-up consisted of regular pap smears according to Dutch guidelines during two years. Successful treatment was defined as no histologic CIN 2/3 diagnosis during follow-up.

Results A total of 84 women were included in the analysis (27 from the imiquimod group and 57 from the LLETZ group). CIN2/3 was diagnosed in one woman (2\%) in LLETZ group and two women in the imiquimod group (7\%), all underwent additional LLETZ treatment $(p=0.26)$. For both entire groups, HPV status at 2 year follow-up was similar. CIN grade at inclusion, HPV status at short term follow-up, age, parity and smoking were not identified as factors associated with successful treatment.

Conclusions Disease recurrence of high-grade CIN two years after successful treatment with imiquimod is infrequent and is not statistically different from LLETZ treatment. This indicates a lasting effectiveness of imiquimod treatment.

\section{EPV238/\#131 IMPACT OF LYMPHADENECTOMY AND INTRAOPERATIVE TUMOR RUPTURE ON SURVIVAL IN EARLY STAGE MUCINOUS OVARIAN CANCERS}

${ }^{1} \mathrm{RS}$ Kim*, ${ }^{2} \mathrm{~A}$ Madariaga, ${ }^{1} \mathrm{~L}$ Hogen, ${ }^{3} \mathrm{D}$ Vicus, ${ }^{3} \mathrm{~A}$ Covens, ${ }^{4} \mathrm{C}$ Parra-Herran, ${ }^{2} \mathrm{~S}$ Lheureux, ${ }^{3} \mathrm{~L}$ Gien. ${ }^{1}$ Princess Margaret Cancer Centre/University of Health Network/Sinai Health Systems, Gynecologic Oncology, Toronto, Canada; ${ }^{2}$ Princess Margaret Cancer Centre/ University of Health Network/Sinai Health Systems, Medical Oncology, Toronto, Canada; ${ }^{3}$ Sunnybrook Health Sciences Centre, Gynecologic Oncology, Toronto, Canada; ${ }^{4}$ Brigham and Woman's Hospital, Pathology, Boston, USA

\subsection{6/ijgc-2021-IGCS.309}

Objectives The aim of the study was to investigate the prognostic significance of lymphadenectomy and intraoperative tumor rupture in patients with apparent stage I mucinous ovarian carcinoma (MOC).

Methods We conducted a retrospective cohort study of MOCs diagnosed between 1999-2019 at two tertiary cancer centers. Pathology was reviewed to rule out metastasis from gastrointestinal tract. Clinicopathologic details, five-year overall survival (OS) and recurrence free survival (RFS) were examined. Cox proportional hazard models were used to determine the association of lymphadenectomy and intraoperative rupture on survival.

Results of 149 with apparent stage I disease, 48 (32\%) had pelvic and/or para-aortic lymphadenectomy, but only 1 patient with grade 2 disease was upstaged due to positive pelvic lymph nodes. Intraoperative rupture was documented in 52 (35\%); these were more likely to have initial surgery performed by a non-gynecologic oncologist $(48 \%$ vs. $11 \%$; $\mathrm{p}<0.001)$. There were 20 recurrences in the cohort $(13 \% ; 9$ grade 1, 6 grade 2,4 grade 3 ), with the vast majority peritoneal (95\%). On multivariable analysis, after adjusting for age, final stage, and use of adjuvant chemotherapy, there was no significant association between intraoperative rupture with OS (HR $2.2(0.6-8.0), \quad p=0.25)$ or RFS (HR 1.3 (0.5-3.3), $\mathrm{p}=0.63)$ or lymphadenectomy with OS (HR 0.9 (0.3-2.8), $\mathrm{p}=0.90)$ or RFS (HR $1.2(0.5-3.0), \mathrm{p}=0.73)$.

Conclusions In apparent stage I MOC, systematic lymphadenectomy has low utility, as few patients are upstaged and recurrence typically occurs in the peritoneum. Furthermore, intraoperative rupture does not independently confer a worse survival.

\section{EPV239/\#263 EVALUATION OF THE IMPACT OF POSTOPERATIVE ADJUVANT THERAPY ON SURVIVAL AND RECURRENCE PATTERNS IN STAGE I-IV UTERINE CARCINOSARCOMA}

1J Mceachron*, 'Y-J Chen, ${ }^{1} \mathrm{~N}$ Zhou, ${ }^{2} \mathrm{C}$ Gorelick, ${ }^{2} \mathrm{M}$ Kanis, ${ }^{1} \mathrm{~J}$ Fehniger, ${ }^{1} \mathrm{YC}$ Lee. ${ }^{1}$ SUNY Downstate Health Sciences University, Gynecologic Oncology, Brooklyn, USA; ${ }^{2}$ New York Presbyterian Brooklyn Methodist Hospital, Gynecologic Oncology, Brooklyn, USA

\subsection{6/ijgc-2021-IGCS.310}

Objectives To evaluate differences in survival and recurrence patterns in stage I-IV uterine carcinosarcoma (UCS) patients treated with surgery followed by adjuvant chemotherapy (CT), radiation (RT) or both (chemoRT).

Methods A multicenter retrospective analysis of patients with surgically staged UCS receiving adjuvant therapy from 2000 to 2019 was conducted. Sites of recurrence were analyzed by adjuvant treatment modality using Pearson's $\chi^{2}$-test. PFS and OS were calculated using Kaplan-Meier estimates. Multivariate analysis (MVA) was performed using Cox proportional hazards model.

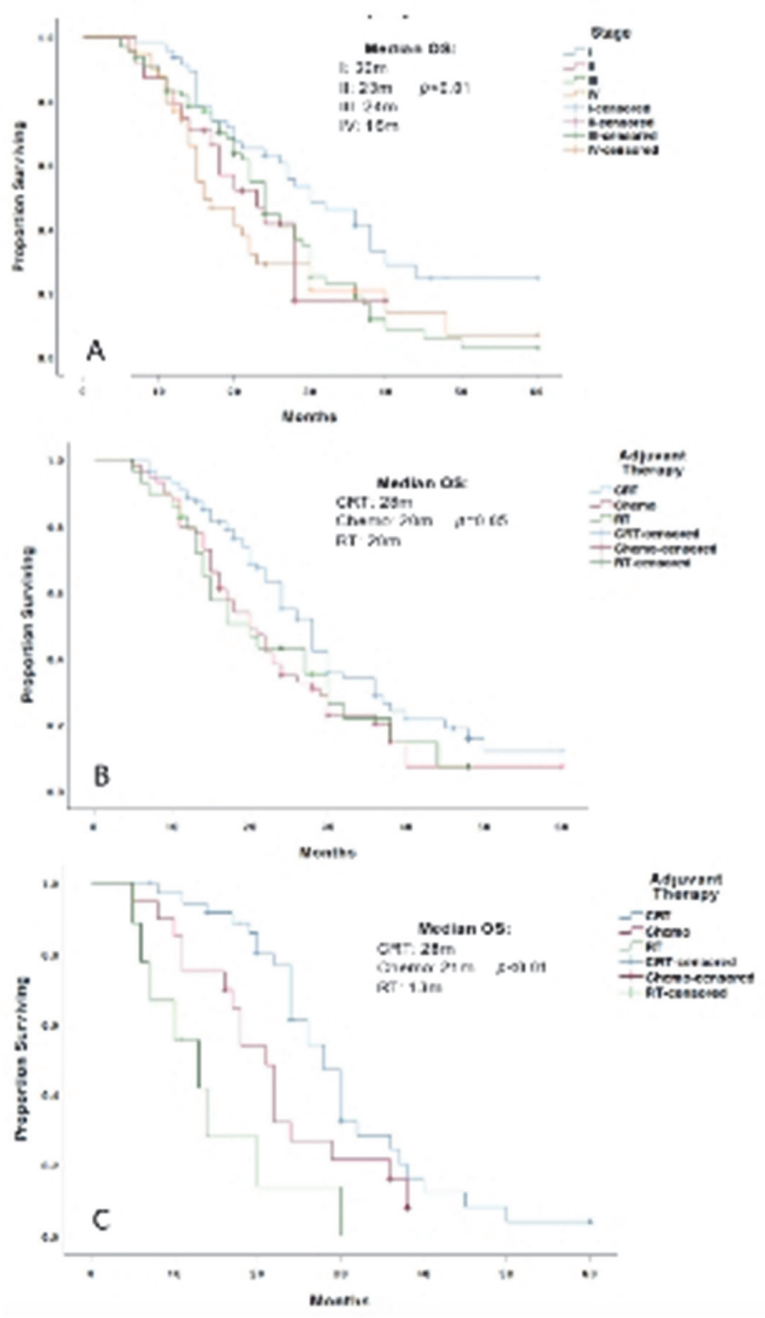

Abstract EPV239/\#263 Figure 1 Overall survival (OS) based on Kaplan-meier estimates

A: OS based on FIGO stage; B: OS of all stages based on the type of adjuvant theraphy; C: OS of stage III disease based on the type of adjuvant theraphy 